www.uoc.edu/idp

ARTÍCULO

\title{
El proceso judicial electrónico y su encaje en el ordenamiento jurídico español: estudio comparado con el proceso electrónico británico
}

\author{
María José Catalán Chamorro \\ Universidad de Córdoba
}

Fecha de presentación: octubre de 2019

Fecha de aceptación: marzo de 2020

Fecha de publicación: junio de 2020

\begin{abstract}
Resumen
España está capacitada para iniciar un proceso judicial electrónico automatizado, similar al implementado en el Reino Unido. En el presente trabajo se expone de manera paralela el análisis del proyecto británico y una propuesta de lege ferenda para la inclusión de esta modalidad en el ordenamiento jurídico español. Esta se iniciaría solamente para reclamaciones de cantidad y procesos monitorios y podría continuar con la resolución electrónica de los procesos por sanciones administrativas de tráfico, finalizando este primer estadio de su desarrollo con la resolución de algunos de los procesos civiles recogidos a través de la Ley de Jurisdicción Voluntaria, incluyendo una propuesta final para habilitar a la ciudadanía para introducir denuncias administrativas o penales en línea. De esta manera, acercaríamos definitivamente el acceso a la justicia a los ciudadanos y ciudadanas, pretendiendo una suerte de autodefensa regulada y garantizada por los jueces y tribunales a la par que cercana a la ciudadanía a través de una plataforma informática segura e intuitiva.
\end{abstract}

\section{Palabras clave}

justicia electrónica, proceso civil electrónico automatizado, jurisdicción voluntaria 


\title{
Electronic process of law and its implementation in the Spanish legal system: a study of comparison with the British electronic process
}

\begin{abstract}
Spain is capable of launching an automated electronic judicial process, similar to the one implemented in the United Kingdom. In the present paper, we present in parallel the analysis of the British project and a proposal of lege ferenda for the inclusion of this modality in the Spanish legal system. The first stage of its development would conclude with the resolution of some of the civil proceedings under the Voluntary Jurisdiction Act, including a final proposal to enable citizens to file administrative or criminal complaints online. In this way, we would definitely bring access to justice closer to citizens, pretending a sort of self-defense regulated and guaranteed by judges and courts as well as close to citizens through a secure and intuitive computer platform.
\end{abstract}

\section{Keywords}

E-Justice, Automated Electronic Civil Procedure, Voluntary Jurisdiction 


\section{Introducción}

El ritmo de la sociedad ha cambiado, casi todo está automatizado, ya no podemos vivir sin las compras, las gestiones administrativas o bancarias en línea, rechazamos cada vez más las colas en oficinas o comercios presenciales. Sin embargo, el Derecho -y sobre todo la Justicia- aún reniega del uso de las nuevas tecnologías. No obstante, en los últimos tiempos nos hemos visto acorralados hacia el uso de estos instrumentos. Ejemplo de ello son LexNET Justicia o los portales Adriano y Minerva, así como las importantes bases de datos o revistas digitales que consultamos los operadores del Derecho para estar al día de las novedades doctrinales y jurisprudenciales.

La evolución social avanza hacia los procedimientos de resolución alternativa de conflictos en línea que tienen cada vez más incidencia en nuestro día a día, principalmente en las reclamaciones de consumo'. Es decir, formas alternativas flexibles de justicia o de tutela efectiva de nuestros derechos que permiten a la ciudadanía acceder a la justicia de una manera sencilla y ágil, en ocasiones sin necesidad de desplazarse, desde su domicilio u oficina, y en cualquier momento del día o de la semana. De resultas, la justicia también se puede adaptar a las necesidades y tiempos de las personas.

Ciertamente, en España vemos como algo muy lejano el proceso electrónico automatizado; sin embargo, como iremos advirtiendo a lo largo del presente trabajo, este está cada vez más cerca, convirtiéndose poco a poco en un camino por el que irremediablemente tendremos que transitar. Por suerte, nuestra sociedad cuenta cada año con un nivel más alto de conocimientos informáticos y la brecha digital está disminuyendo año a año, tal y como muestran las encuestas que analizaremos más adelante $^{2}$. Todo ello unido al esfuerzo legislativo que se está haciendo por parte de las Administraciones públicas para informatizar y facilitar el acceso a estos medios electrónicos a la ciudadanía a través de la firma digital.

\section{Origen de la e-Justicia en España}

Si tuviésemos que establecer un hito relevante en la legislación española a partir del cual arranca este proceso de automatización de la Administración de Justicia, sería la Ley 18/2011, de 5 de julio, reguladora del uso de las tecnologías de la información y la comunicación en la Administración de Justicia ${ }^{3}$. Este texto legislativo estableció las bases definitivas de la actual plataforma LexNET Justicia.

Sin embargo, esta plataforma se pondría en funcionamiento definitivamente con la entrada en vigor del Real Decreto 1065/2015, de 27 de noviembre, sobre comunicaciones electrónicas en la Administración de Justicia en el ámbito territorial del Ministerio de Justicia y por el que se regula el sistema LexNET ${ }^{4}$, en vigor desde el 1 de enero de 2016, amén de las sucesivas actualizaciones. La última actualización del sistema LexNET 4.18 implementada el 2 de marzo de 2020 crea nuevas funcionalidades y mejoras, además de la actualización de septiembre de 2019 que reforzó su seguridad y agilidad en la gestión de los archivos, y donde se mejoraron los canales de comunicación a través de la puesta en marcha de una dirección de correo electrónico, una cuenta en la red social Twitter y un teléfono para la información y soporte de los usuarios. Estos canales de comunicación eran muy necesarios desde la entrada en funcionamiento de LexNET y llegan con cierto retraso.

Ese primer hito legislativo mencionado marcó definitivamente el futuro de la justicia electrónica en España, ya que dentro del anexo de definiciones de

1. Solo en la plataforma ODR de consumo creada por la Comisión Europea se han registrado más de 131.136 reclamaciones en su cuarto año de funcionamiento, de las que España ha realizado 11.959. Véase: https://ec.europa.eu/consumers/odr/main/?event=main.statistics.show [Fecha de consulta: 13 de marzo de 2020].

2. Véase: Instituto Nacional de Estadística (2018). Población que usa Internet (en los últimos tres meses). Tipo de actividades realizadas por Internet: https://www.ine.es/ss/Satellite?L=es_ES\&c=INESeccion_C\&cid=1259925528782\&p=1254735110672\&pagename=ProductosYServ icios\%2FPYSLayout [Fecha de consulta: 13 de marzo de 2020].

3. BOE núm. 160, de 6 de julio de 2011.

4. BOE núm. 287, de 1 de diciembre de 2015. 
la Ley 18/2011, de 5 de julio, se fue mucho más allá, estableciendo el significado del concepto «actuación judicial automatizada», del que se dice que es aquella actuación judicial producida por un sistema de información adecuadamente programado sin necesidad de intervención de una persona física en cada caso singular (Bueno de Mata, 2015, págs. 4-6). Además, en el artículo 42 del citado texto se decreta la necesidad de establecer, en los casos donde se ejecute la actuación judicial automatizada, un Comité técnico estatal de la Administración judicial electrónica, así como indicadores de gestión que se establezcan por la Comisión $\mathrm{Na}$ cional de Estadística Judicial y el Comité técnico estatal de la Administración judicial electrónica, cada uno en el ámbito de sus competencias (Palomar Olmeda, 2012, pág. 481).

Esta previsión legal incluye desde la producción de actos de trámite o resolutorios de procedimientos, hasta meros actos de comunicación. De este modo, muy acertadamente, el legislador establecía un marco amplio para las legislaciones venideras. Tanto es así que, acogiéndonos a este marco, podríamos implantar un sistema de iniciación electrónica de los procesos -sobre todo de los civiles- sin necesidad de modificar o crear un nuevo amparo legal.

\section{El proyecto de tribunales digitales en el Reino Unido y su encaje en la jurisdicción española}

En el presente apartado analizaremos el proyecto en el que el Reino Unido Ileva trabajando muy activamente desde 2016. Hoy, cuatro años después, ya se están iniciando algunos procesos judiciales en varias jurisdicciones diferentes y sobre temáticas concretas íntegramente a través de internet. Este proyecto está basado en el caso de éxito del Tribunal de Resolución Civil de Canadá en línea -Canadian Civil Resolution Tribunal-5, que lleva operando con total normalidad ocho años. En este país solo se ha visto incrementada la litigiosidad de este orden jurisdiccional en un $1 \%$ y se ha denotado que un $45 \%$ de sus usuarios utili- zan esta plataforma fuera del horario habitual de los tribunales físicos (Slater, 2017). Así las cosas, lo que en un principio nació como un sistema ágil para dar respuesta a las reclamaciones de cantidad inferiores a las 10.000 libras esterlinas, hoy se extiende hasta divorcios, materia testamentaria o incluso denuncias penales.

De este modo, siguiendo un sistema comparado y conociendo la implantación de estos tribunales digitales en el Reino Unido, podemos esbozar algunas instituciones digitales que podríamos fácilmente implantar en España a fin de facilitar el acceso a la justicia de la ciudadanía a través del medio digital.

No obstante, tal y como indicábamos más arriba, el programa de modernización de la justicia británico va más allá de la justicia civil, incluyendo denuncias sobre presuntos delitos o asuntos sobre Derecho de familia, materias en definitiva a las que en principio no se aspiraría en el ordenamiento jurídico español debido a la especial protección que deben tener los menores en asuntos de familia y los denunciados en el ámbito penal, pues, a través del medio electrónico, se podrían infringir principios y garantías básicas del proceso. Aunque -a tenor de los vertiginosos avances tecnológicos- en algunos años probablemente no podremos renunciar a tramitar casi ningún tipo de controversia vía internet.

\subsection{Identificación del actor}

Una de las cuestiones que nos planteamos a priori en España, a la vista de la gran problemática existente con casos de suplantación de identidad a través de internet, es cómo estar seguros en la identificación de un actor o de un demandado si no se han personado en un juzgado para identificarse. Pues bien, en el caso británico, no es necesaria ni tan siquiera la identificación mediante firma digital para acreditarse e iniciar la solicitud de resolución por parte de un órgano judicial. La plataforma electrónica no solo permite efectuar la solicitud de tutela, sino también hacer un seguimiento de la gestión de los casos, todo en línea, además de la posibilidad de celebrar audiencias con el juez a través

5. Ver en: https://civilresolutionbc.ca/about-the-crt/ [Fecha de consulta: 4 de octubre de 2019] 
de videoconferencia e incluso recibir la resolución judicial en el buzón electrónico particular6. $Y$ todo ello sin necesidad de utilizar ningún tipo de identificación oficial ante la Administración de Justicia británica. Simplemente a partir de un registro a través de un email y de una contraseña, podemos iniciar, por ejemplo, nuestra reclamación de cantidad en el Reino Unido, donde se disuade la posible suplantación de identidad por la necesidad de pago de una tasa para dar entrada a las reclamaciones

En España, para cualquier trámite con la Administración pública se requiere del sistema de firma o certificado digital para asegurar la identificación auténtica del ciudadano o ciudadana. Así las cosas, a fin de obtener dicho certificado es precisa la personación física e identificación por parte de un trabajador público para recibir este archivo electrónico que le permita este acceso. En la actualidad este sistema ya es utilizado en nuestro país para los procedimientos de resolución de reclamaciones de consumo en línea a través de la ins titución pública «Arbitraje de Consumo» en casi todas las comunidades autónomas?

\subsection{Sin necesidad de abogado ni de procurador}

El impulso de la Administración británica -al igual que el de la española- está implementándose en la dirección de la autorrepresentación y la autodefensa. Este servicio, como nuestra Ley 15/2015, de 2 de julio, de Jurisdicción Voluntaria ${ }^{8}$, pretende fomentar los procesos sin necesidad de representación legal, lo que se ha denominado litigants in person -y reconocido a través de las siglas LIP- para las reclamaciones civiles de escasa y mediana cuantía (Cortés y Takagi, 2019). De esta manera, la justicia se viste de plataforma virtual, resultando así más accesible y amigable para la ciudadanía. Tal y como señaló a propósito de esta reforma el Lord Chief Justice, lo importante es que «la tecnología sea nuestra servidora, no nuestra ama, y ofrezca a nuestros tribunales la posibilidad de resolver las disputas más rápidamente y menos costosamente» (Burnett, 2018).

Esta tecnología puede venir a paliar los altos costes procesales existentes en nuestro sistema procesal civil, donde la quota litis supone un alto porcentaje dentro de las reclamaciones de cantidad o de los conflictos de escasa cuantía, obteniendo como resultado que la inmensa mayoría de estos asuntos quedan fuera del real y efectivo acceso a la justicia para el ciudadano medio de nuestro país, y, por ende, muestran una debilidad del sistema de justicia.

En este sentido se pronunciaba en su informe el británico lord Briggs, quien observó que: «La debilidad más omnipresente y de hecho más chocante de nuestra corte civil es que no proporcionan un acceso razonable a la justicia para los individuos ordinarios» (Briggs, 2016); y es que, ciertamente, para una mayoría de la población los tribunales de justicia quedan muy lejos de sus expectativas cuando lo que intentan reclamar son cantidades de escasa cuantía, debido al elevado porcentaje de quota litis que comportan los honorarios de los abogados y procuradores. En España, esta cuestión ha intentado ser paliada con normas como la reforma de la Ley de Enjuiciamiento Civil a través de la Ley 4/2011, de 24 de marzo $^{9}$, y de la Ley 42/2015, de 5 de octubre $^{10}$, por la que los litigantes podrán comparecer por sí mismos, es decir, sin necesidad de abogado ni procurador, en los juicios verbales cuya determinación se haya efectuado por razón de la cuantía y esta no exceda de 2.000 euros, así como en la petición inicial del procedimiento monitorio y hasta el momento en el que se plantee oposición. Aunque el espaldarazo definitivo ha venido dado por los diecinueve tipos de expedientes de jurisdicción voluntaria que la Ley 15/2015 permite realizar sin necesidad de abogado ni procurador. Sin embargo, nos encontramos con una problemática colateral como es el desconocimiento casi pleno que tiene la ciudadanía de estos instrumentos y de cómo obtener

6. Ver en: https://www.gov.uk/make-money-claim [Fecha de consulta: 4 de octubre de 2019].

7. Ejemplo de ello son: Andalucía https://ws231.juntadeandalucia.es/eadministracion/menu.do, Comunidad Madrid https://gestionesytramites.madrid.org/, Cataluña https://juntarbitral.bcn.cat/es/solicitud-de-arbitraje o Comunidad Valenciana https://www.gva.es/es/inicio/ procedimientos?id_proc=2290\&version=amp [Fecha de consulta: 4 de octubre de 2019].

8. BOE núm. 158, de 3 de julio de 2015.

9. BOE núm. 72 , de 25 de marzo de 2011.

10. BOE núm. 239, de 6 de octubre de 2015 
una tutela judicial efectiva sin necesidad de acudir a profesionales del Derecho. Para la mayoría de ciudadanos y ciudadanas, los juzgados suelen ser instituciones lejanas en las que no creen poder actuar en su propio nombre. No obstante, es posible que, si establecemos una plataforma virtual suficientemente intuitiva para la ciudadanía, esta percepción pueda cambiar.

Si analizamos el conocimiento medio del ciudadano español para relacionarse telemáticamente con la Administración pública no es excesivamente alarmante, ya que un $47,2 \%$ de los encuestados afirma haber enviado formularios cumplimentados a alguna Administración o servicio público a través de la red en los últimos doce meses y un 65,4\% asegura haber contactado o interactuado con las Administraciones o servicios públicos vía internet por motivos particulares también en el mismo período de tiempo. Estas cifras aumentan hasta un 49\% y un $69,6 \%$ respectivamente si los encuestados tienen estudios terminados de segunda etapa de educación secundaria y siguen aumentando a mayor formación de la muestra consultada". Así las cosas, podemos considerar que aproximadamente el 50\% de la población española estaría capacitada para seguir un proceso judicial civil, de reclamación de cantidad sencillo a través del medio en línea y donde el ciudadano se represente y se defienda a sí mismo.

\section{Inconvenientes en la digitalización de la justicia}

A pesar de todos los beneficios ya expuestos en el presente trabajo, debemos ser realistas y contar con los diferentes inconvenientes que podrían surgir con la puesta en marcha de estos tribunales electrónicos.

En primer lugar, es cierto que podría suponer un hándicap para el Estado la inversión inicial que a priori necesita esta nueva tecnología para ponerse en marcha, sobre todo con las suficientes garantías que requiere la tutela judicial efectiva del artículo 24 de la Constitución, unido a los posibles problemas de seguridad e identificación de los individuos que se podrían plantear. No obstante, en nuestro país tenemos la experiencia positiva de las declaraciones de la renta, que se realizan mayoritariamente a través de internet y que no provocan, generalmente, ningún tipo de problemática acerca de las garantías, seguridad del sistema o identidad de los declarantes.

Y, en segundo lugar, también podría suponer un hándicap, en este caso para la ciudadanía española, la introducción de tasas para la utilización de estas plataformas telemáticas. Este sistema, a pesar de ser más económico que la quota litis, precisa de un sistema de tasas que de alguna manera sustente y dé seriedad a las acciones legales que se inicien en la plataforma.

Las tasas que plantea la plataforma británica son económicas: parten de 25 libras para reclamaciones de hasta 300 libras y llegan hasta un máximo de 410 libras de tasas si la reclamación es desde 5.000,01 hasta 10.000 libras $^{12}$. En la propia plataforma también se introducen a modo comparativo la tasa del mismo tipo de reclamación en papel o por medio de la modalidad física. Con todo, estas tasas serán menos costosas, ya que a través de esta vía telemática no será necesaria la asistencia de ningún profesional del Derecho, debido a que la plataforma sería suficientemente intuitiva $y$, además, establecería un sistema de asistencia al reclamante o reclamado tanto vía telefónica como vía correo electrónico para ayudarle a resolver sus dudas.

No obstante, es necesario apuntar que esta tasa no es cerrada para todo el proceso, sino que en cada una de las instancias de este que se superen sin obtener la solución a la disputa se cobrará una tasa extra antes de acceder, por ejemplo, a la vista con el juez tras haberse intentado por este una solución amistosa en una audiencia previa o para recurrir la apelación de la decisión obtenida ${ }^{13}$

11. Fuente: Encuesta del INE sobre formas de contacto o interacción con las Administraciones o servicios públicos por Internet, por motivos particulares, en los últimos doce meses por características socioeconómicas y tipo de acción: https://www.ine.es/jaxi/Datos.htm?path=/ t25/p450/base_2011/a2018/I0/\&file=04014.px [Fecha de consulta: 13 de marzo de 2020].

12. Disponible en: https://www.gov.uk/make-court-claim-for-money/court-fees [Fecha de consulta: 13 de marzo de 2020].

13. Disponible en: https://www.gov.uk/make-court-claim-for-money/court-fees [Fecha de consulta: 18 de marzo de 2020 
Sin embargo, a pesar de los altos costes iniciales que tendría este sistema para el Estado y la adaptación de la ciudadanía a un nuevo sistema de tasas judiciales para la resolución de sus disputas, creemos que sigue siendo conveniente la implantación de este sistema de justicia civil electrónica en nuestro país.

\section{Procesos judiciales afines para la digitalización}

Vista la experiencia de otros países que ya llevan años desempeñando procesos electrónicos, debemos entender que esto ha sido un procedimiento adaptativo y evolutivo, iniciándose con algunos trámites electrónicos, continuando con algunos procesos e incluyendo paulatinamente una variedad mayor de materias enjuiciables electrónicamente con el paso de los años. Por ello, del gran abanico de tipos de procesos que tenemos en nuestro país para solventar los conflictos, podríamos comenzar con los procesos civiles de escasa cuantía y proseguir con la iniciación de procesos monitorios, reclamaciones por sanciones administrativas de tráfico y quizá la digitalización de algunos expedientes de jurisdicción voluntaria.

\subsection{Reclamaciones de escasa cuantía}

Las reclamaciones de escasa cuantía son fácilmente operables a través de la plataforma web $^{14}$ que el Gobierno británico ha puesto en marcha. Se trata de un sistema sencillo, que recuerda mucho a la plataforma web $\mathrm{ODR}^{15}$ de reclamaciones de consumo europea implementada por la Comisión Europea en febrero de 2015. Para ello, el Reino Unido ha aprovechado los procesos monitorios de reclamación de cantidad -que ya se podían llevar a cabo sin asistencia letrada- y han abierto la posibilidad de efectuar estos procedimientos monitorios en línea para cantidades determinadas e inferiores a 10.000 libras. Este proyecto primigenio fue el Civil Money Claims Online (su acrónimo, CMCO) y, aunque su límite actual es de 10.000 libras esterlinas, se espera que pronto se incremente a 25.000, si bien excluye los casos más complejos, como pueden ser los de lesiones personales. La previsión estimada por el Ejecutivo británico ha sido que la inmensa mayoría de las demandas civiles por reclamación de cantidad corresponderán a este sistema, ya que solo el 10\% de todas las demandas civiles superan las 25.000 libras esterlinas.

El proyecto piloto de la $\mathrm{CMCO}$, que comenzó en marzo de 2018, ha registrado unos magníficos resultados, pues en junio de 2019 había tramitado más de 70.000 casos, con una tasa de satisfacción de los usuarios de estos tribunales del 90\% (House of Commons Select Justice Committee, 2019).

\subsection{Tribunales telemáticos para las sanciones de tráfico}

Además de procesos civiles sencillos de reclamación de cantidades, existe otra institución británica que podríamos poner en marcha en nuestro país y que satisfará a la ciudadanía española: es la figura del Traffic Penalty Tribunal -Tribunal de Sanciones de Tráfico-, que se ocupa de las apelaciones contra las multas de tráfico impuestas por las autoridades locales y proporciona un servicio en línea que ofrece audiencias telefónicas y virtuales, además de permitir a las partes cargar las pruebas en la plataforma y contar con un equipo de asistencia digital (Sheppard, 2018).

Actualmente, en España, para recurrir cualquier multa de tráfico se puede interponer un escrito de alegaciones y, posteriormente, un recurso de reposición. Si ambos no han sido satisfactorios para el ciudadano, a este solo le queda acceder a la jurisdicción contenciosoadministrativa. Este orden jurisdiccional es el encargado de llevar a cabo estos procesos, que, a pesar de ser relativamente sencillos y ágiles, suponen un alto coste tanto para la ciudadanía como para el Estado. El ciudadano recurrente deberá ser defendido y representado por abogado y procurador respectivamente, amén del riesgo al que se somete de ser condenado a pagar to-

14. Disponible en: https://www.gov.uk/make-money-claim [Fecha de consulta: 13 de marzo de 2020].

15. Disponible en: https://ec.europa.eu/consumers/odr/main/index.cfm?event=main.home2.show\&lng=ES [Fecha de consulta: 13 de marzo de 2020]. 
das las costas del proceso tras la entrada en vigor de la Ley 37/2011, de 10 de octubre, de medidas de agilización procesal $^{16}$. Y, por otro lado, el Estado deberá soportar los costes que producen desde las vistas o audiencias que se han de celebrar de manera presencial, hasta la representación de los intereses del Estado a través de la Abogacía del Estado, también presencialmente. Razones de peso para dar la posibilidad al ciudadano de realizar este tipo de procedimientos a partir de una plataforma telemática, de manera eminentemente escrita, con la prueba en soporte documental y, si fuese precisa, la celebración de la vista por medio de videoconferencia, así como establecer como no preceptiva la asistencia de abogado ni de procurador para aquellas multas de tráfico administrativas inferiores a 2.000 euros, asimilando esta situación a las reclamaciones de cantidad entre privados recogidas en la Ley de Enjuiciamiento Civil. Y como es evidente quedarían fuera de estos tribunales virtuales aquellas sanciones penales derivadas de la conducción imprudente o dolosa de los ciudadanos.

\subsection{Procesos recogidos en la Ley de Jurisdicción Voluntaria}

Como indicábamos en apartados anteriores, para el ejercicio de los expedientes de jurisdicción voluntaria no es preceptiva la asistencia de abogado y procurador en expedientes relativos a la autorización o aprobación judicial del reconocimiento de la filiación no matrimonial; la habilitación para comparecer en juicio y el nombramiento de defensor judicial; la adopción; para la tutela, la curatela y la guarda de hecho, salvo para la remoción del tutor o curador; o la concesión judicial de la emancipación y del beneficio de la mayoría de edad, entre otros. Si bien es cierto que no todos los expedientes son aptos para la tramitación electrónica, porque, a pesar de que exista la posibilidad de celebrar vistas a través de videoconferencia, sobre todo en procesos donde intervenga el Ministerio Fiscal en defensa del interés superior de los menores o incapaces será necesario la presencia y examen de los mismos.
No obstante, existen otros muchos expedientes que son simples trámites administrativos que pueden ser perfectamente iniciados y tramitados a través de una plataforma electrónica desde el domicilio de los ciudadanos con la suficiente seguridad y credibilidad que otorga la firma digital para efectuar cualquier tipo de acto administrativo. Posteriormente se requerirá la actuación del juez o del letrado de la Administración de Justicia, según el caso, en atención a la autoridad que el titular de la potestad jurisdiccional merece como intérprete definitivo de la ley, imparcial, independiente y esencialmente desinteresado en los asuntos que ante ella se dilucidan. En estos casos de expedientes electrónicos sería recomendable retirar la potestad de estos expedientes a notarios y registradores de la propiedad y mercantiles ${ }^{17}$ para, así, aumentar las garantías y la confianza en este sistema electrónico bidireccional exclusivo entre la Administración de Justicia y el conjunto de la ciudadanía.

\section{El funcionamiento interno de la plataforma británica}

\subsection{Primera fase}

En primer lugar, la plataforma web de reclamaciones de escasa cuantía británica realiza un test de idoneidad a fin de adaptar esta herramienta al usuario y comprobar la jurisdicción de los tribunales británicos, la capacidad de los participantes, los posibles litisconsorcios o la correcta representación de las partes. Sobre la base de este test, donde se preguntan cuestiones sencillas como si la persona reclamante y reclamada están domiciliadas en territorio británico y ambos son mayores de edad, o si la reclamación se dirige contra una o varias personas y si estas son personas físicas o jurídicas, así como si se realiza la reclamación en nombre propio o en representación de un cliente o una organización. Además, la plataforma requiere cerciorarse de que la reclamación cumple con el límite máximo monetario de

16. BOE núm. 245, de 11 de octubre de 2011, por el que se modificó el artículo 139 de la Ley 29/1998, de 13 de julio, reguladora de la Jurisdicción Contencioso-Administrativa.

17. Preámbulo de la Ley de Jurisdicción Voluntaria: «Como regla general, los expedientes de jurisdicción voluntaria en materia de personas y de familia, y también alguno de los expedientes en materia mercantil y de Derecho de obligaciones y sucesorio que no se encomiendan a Secretarios judiciales, Notarios o Registradores». 
las 10.000 libras, así como de si la persona reclamante tendrá problemas para pagar la tasa que comentábamos en apartados anteriores.

Una vez el ciudadano haya realizado con éxito el test de idoneidad, donde la tipología de la reclamación encaje con los supuestos que el sistema permite suscribir, se tendrá que identificar en una cuenta de usuario. A través de esta cuenta de usuario deberá aceptar un consentimiento informado donde se le indica al reclamante que debe haber intentado solucionar el conflicto en persona con su contraparte, así como considerar la opción de la mediación como posible y factible para este tipo de casuísticas. Asimismo, también tendrá que aceptar un consentimiento donde se le indica que si a posteriori quiere cambiar algún dato de la reclamación deberá pagar una tasa extra.

Aceptados estos consentimientos podrá iniciar la reclamación introduciendo los datos del reclamante y de su contraparte; la cantidad por la que desea reclamar, y el resto de detalles de la reclamación que el reclamante quiera que se tengan en cuenta. No obstante, para la remisión de esta reclamación al órgano competente será necesario efectuar con carácter previo el pago de la tasa a través de la plataforma web.

Actualmente, esta herramienta está siendo transferida al personal de la Administración de Justicia británica para la toma de decisiones en una primera instancia o fase. De esta manera se pone en valor la agilidad y la rapidez en las respuestas, aunque se pone en duda la transcendencia en sus decisiones, así como la idoneidad de estos profesionales para ejercer tal nivel de poder decisional. La Administración británica los ha denominado «officers case lawyers» para destacar sus conocimientos en el ámbito jurídico (Cortés y Takagi, 2019). No obstante, esta cuestión es perfectamente adaptable al sistema judicial español ya que en nuestro país es el letrado de la Administración de Justicia el encargado en primera instancia de realizar la mediación previa en los procesos, en virtud de la atribución competencial que les confiere el artículo 456.6.e de la LOPJ.
Una vez suscrita la reclamación y la contestación a la misma por parte de la contraparte podemos encontrarnos diferentes escenarios. Bien que la contraparte realice el pago de la cantidad reclamada y ahí finalizaría el proceso, o bien que la contraparte no esté de acuerdo con la reclamación por diferentes circunstancias, como por ejemplo que el reclamado responda que no debe ninguna cantidad al reclamante; que esta cantidad que le reclama no se ajusta a la realidad; que no está de acuerdo con la utilización de este procedimiento y requiere otro físico ${ }^{18}$ y no en línea, siendo incluso posible que ni siquiera conteste a la reclamación.

En el caso de que la contraparte se persone se invitará a las partes a intercambiar información en línea y a buscar por sí mismas una solución anticipada mediante ofertas cruzadas elaboradas por el propio sistema electrónico.

Así las cosas, en este primer nivel o etapa se sitúa una autoevaluación efectuada a través de un test en línea y en el que la plataforma ya sugiere ofertas para proponer a la contraparte, seguida de una negociación entre ambas partes. Si en solitario no son capaces de llegar a un acuerdo, a esta negociación le seguirá una etapa de facilitación en la que un tercero neutral ayuda a los litigantes a resolver su litigio.

Este tercero neutral será encarnado por los antes comentados «officers case lawyers». Estos asesores jurídicos son personal judicial legalmente cualificado y capacitado que puede ayudar a las partes en la gestión de su caso y a llegar a un acuerdo a partir de un sistema de resolución alternativo de disputas vía internet o de una mediación telefónica. Es necesario apuntar que las decisiones tomadas por los «officers case lawyers» son siempre supervisadas por los jueces, de modo que lo que aquellos realizan no es más que la facilitación al entendimiento de las partes y promover propuestas, que legalmente son aceptables, para la resolución en cada uno de los conflictos.

18. No obstante, para intentar paliar la brecha digital y los diferentes problemas o dudas técnicas que puedan surgir, las partes disponen de apoyo a través de un chat electrónico y una línea telefónica, así como una nueva aplicación incluida dentro de la plataforma Ilamada Assisted Digital, diseñada para ayudar a los usuarios de los tribunales con poca competencia digital. 
Las mediaciones que están autorizados a realizar el personal de la Administración de Justicia británica ${ }^{19}$ es similar a las conciliaciones que en España pueden implementar los letrados de la Administración de Justicia de los juzgados de primera instancia o de lo mercantil para cuantías superiores a los 6.000 euros y los jueces de paz si es inferior a dicha cantidad. La competencia territorial en este caso será siempre la del domicilio del requerido en virtud del artículo 140 de la Ley 15/2015, de 2 de julio, de la Jurisdicción Voluntaria. En el caso español, la conciliación no tiene vertiente en línea, aunque podría tenerla sobre la base de una estructura similar a la británica. Si bien es cierto que nuestra Ley de Jurisdicción Voluntaria, con muy buen criterio, elimina la posibilidad de someter a este tipo de procedimientos a los interesados que sean menores o personas con capacidad modificada judicialmente para la libre administración de sus bienes, ya que en estos deberá ser parte también el Ministerio Fiscal, por existir intereses superiores necesitados de protección.

\subsubsection{Inteligencia artificial y ODR: ¿ventaja o desven- taja?}

En esta primera fase, la plataforma británica trabaja activamente para evitar la imposición de la decisión por un tercero, ayudando a las partes en la búsqueda de una solución común y autocompositiva. De este modo, se apuesta, al igual que en otras experiencias previas de justicia en línea, por la resolución alternativa del conflicto -como la exitosa herramienta de eBay ${ }^{20}$ para solventar desavenencias entre sus clientes-. Sin embargo, es posible que los creadores de esta plataforma estén almacenando todos los casos, sus parámetros, ofertas, contraofertas y modos de acuerdo en un sistema de big data (o macrodatos) para a posteriori, a través de la inteligencia artificial, mejorar los sistemas de oferta, de soluciones más adaptadas y casos de éxito para las partes, sin necesidad de que un tercero acabe imponiendo su voluntad.
En definitiva, se trataría de buscar una especie de programa especialista en crear un diagnóstico de cada problemática, basándose en las experiencias previas resueltas exitosamente en esta primera fase de resolución de los conflictos. Esta herramienta entraría dentro del ámbito de los Online Dispute Resolution (en adelante ODR), si bien no podemos aclarar la tipología de este ODR, es decir si funciona a través de mediación, conciliación o arbitraje, o forma parte de los Ilamados ODR híbridos, los cuales aprovechan las bondades de los métodos autocompositivos y adjudicativos para crear nuevas modalidades con personalidad propia, dando lugar a diferentes figuras como pueden ser los med-arb, co-med-arb o multi-step-wise-men (Vilalta Nicuesa, 2013, págs. 65-68).

Sin embargo, es cada vez más evidente que la alimentación de estas bases de datos de los sistemas de resolución alternativa de conflictos con objeto de que, a través de las experiencias previas, se creen algoritmos que nos den la respuesta más ajustada a nuestro conflicto particular, es a partes iguales tan beneficiosa, debido a la agilidad que nos proporciona, como contraproducente, a causa de la valiosa información personal que le regalamos con cada una de nuestras decisiones al propio sistema.

\subsubsection{La especialización de los juzgadores}

Este sistema de reconocimiento electrónico inteligente de conflictos podría asimismo derivar cada casuística o problemática a los jueces especialistas en las mismas (Cortés, 2018, págs. 103-121). No obstante, esto podría chocar con el principio de un juez ordinario predeterminado por la ley, a menos que se creen juzgados telemáticos especializados en cada tipo de problemática. El sistema podría determinar, según los datos introducidos y marcados por el reclamante, la competencia objetiva y territorial del conflicto, así como el juez que por turno se establezca competente, en atención a las normas de reparto del juzgado o tribunal competente -previamente introducidas en el sistema informático-.

19. Ver más en: Courts and Tribunals (Judiciary and Functions of Staff) Bill Factsheet: Authorised Court and Tribunal Staff-legal advice and judicial functions: https://www.gov.uk/government/publications/courts-and-tribunals-judiciary-and-functions-of-staff-bill [Fecha de consulta: 13 de marzo de 2020].

20.Podemos considerar el ODR iniciado por las empresas eBay y PayPal en 1999 como primer hito más importante de la historia del ODR de consumo. Ver más sobre el fenómeno ODR de eBay en: C. Rule (2008); y E. Katsh, J. Rifkin y A. Gaitenby (2000). 
Al mismo tiempo el sistema podría efectuar un primer «cribado» relativo a la legitimación de este reclamante y determinar la competencia subjetiva del caso.

\subsection{Segunda fase}

Pasado el período de facilitación o conciliación, y no habiendo lugar a un acuerdo entre las partes, será el momento en que el «officer case lawyer» tome una decisión sobre la resolución del expediente. No obstante, es necesario referenciar que, si bien hasta el momento los «officers case lawyers» tienen potestad para imponer una respuesta final a las reclamaciones monetarias inferiores a 300 libras esterlinas, las partes siempre podrán requerir la revisión de la decisión por el juez. Como indicábamos anteriormente los casos asignados a los «officers» son los que le corresponden al juez con el que trabajan regularmente, lo que permite que ambos mantengan una comunicación fluida acerca de los expedientes que se están llevando a cabo. Sin embargo, estos «officers» no están autorizados para dar consejo jurídico a las partes y solo pueden asesorar en el ámbito procesal de la resolución del conflicto indicando o direccionando el mismo.

Finalmente, si la labor del «officer» no ha conseguido resolver el conflicto entre las partes y el valor de la reclamación es superior a 300 libras será entonces cuando un juez adjudique una decisión con respecto al conflicto. En esta etapa final, un juez decide sobre las reclamaciones basándose en los escritos presentados por las partes y en una audiencia que se espera se ofrezca a las partes a través de medios electrónicos y permita la inmediación necesaria por medio de teléfono o videoconferencia.

\subsubsection{La vista}

Así las cosas, la fase más en el aire de todo este proceso en línea británico para reclamaciones civiles es la vista. En este sistema está permitido que ambas partes comparezcan mediante videoconferencia, pero aún quedan incógnitas por resolver: por ejemplo, si las partes que asisten a la misma lo harán físicamente desde cualquier sede judicial o institucional habilitada por la Administración de Justicia -como es el caso actual de España-21, o si será posible realizarla incluso desde el domicilio o lugar de trabajo de las partes con una simple autenticación del usuario. Sin lugar a dudas, España todavía necesitará que las partes asistan a estas vistas desde una sede judicial o policial para dar ese halo de oficialidad necesario en nuestros procesos judiciales, sin perjuicio de que en un futuro se permita a las partes asistir a estas vistas desde cualquier otro lugar físico a su elección.

\subsubsection{La publicidad del proceso}

Otra cuestión controvertida que surge a la luz de las vistas es el aseguramiento de la publicidad del proceso, es decir, cómo hacemos para que cualquier ciudadano o ciudadana pueda asistir a este tipo de vistas en línea donde cada parte se encuentra deslocalizada de la sede judicial en la que se celebra. Sin duda, la solución electrónica es factible, ya que se podrían habilitar pantallas en los juzgados para la visualización de estos procesos de una forma similar a como actúan los tablones edictales, tal y como ya se ha previsto para el caso británico.

\section{La denuncia en línea ¿es posible?}

Todo este sistema hasta aquí definido tiene total y absoluta cabida en el ordenamiento jurídico procesal español sin crear ningún conflicto importante entre sus normas. En el presente trabajo nos hemos centrado en las posibilidades que el proceso electrónico automatizado nos proporciona en el ámbito civil, aunque también es interesante esbozar las utilidades que nos puede ofrecer en el de las denuncias públicas para informar sobre posibles irregularidades o incluso delitos que los ciudadanos presencian a diario.

En ocasiones, la interposición de una denuncia ante cualquier cuerpo de seguridad del Estado o Adminis-

21. En virtud del artículo 229.3 de la LOPJ. 
tración pública lleva aparejada para los denunciantes no agraviados un compromiso ético y social, además de una cesión importante de tiempo, ya que el desplazamiento hasta la comisaría o Administración pública y la declaración para la denuncia suele conllevar más tiempo del que en la vida cotidiana dispone la ciudadanía tras sus obligaciones laborales y familiares.

Por ello, del mismo modo que existe una aplicación informática para el aviso de la perpetración de determinados delitos y la personación de la policía en el lugar donde se esté cometiendo el mismo, igualmente sería posible que, a través del sistema de autenticación comentado con firma digital, se pudieran emprender denuncias en línea. Si bien, en caso de que la policía o Administración requiriese más datos o aclaraciones sobre lo dispuesto en la misma, estas instancias podrán entrevistar al denunciante vía telefónica o citarlo en sus dependencias con la finalidad de esclarecer los hechos denunciados. De resultas, se podría abrir línea directa con diversas Administraciones responsables de la inspección en diferentes sectores -laboral, sanitario, alimentario, educativo, de la hacienda pública, etc.- a fin de alertarlas de posibles irregularidades conocidas por los denunciantes. No obstante, estos últimos tendrán que marcar una declaración jurada y ser advertidos de las sanciones en caso de denuncia falsa. 


\section{Referencias bibliográficas}

BRIGGS, L. J. (2016). Civil Courts Structure Review: Final Report, pág. 28 [en línea] https://www.judiciary.uk/wp-content/uploads/2016/07/civil-courts-structure-review-final-report-jul-16-final-1.pdf [Fecha de consulta: 13 de marzo de 2020].

BUENO DE MATA, F. (2015). «Mediación electrónica e inteligencia artificial». Actualidad Civil, núm. 1, págs. 4-6.

BURNETT, I. (Lord Chief Of Justice) (2018). Keynote Speech at the First International Forum on Online Courts the Cutting Edge of Digital Reform [en línea]. https://www.judiciary.uk/wp-content/ uploads/2018/12/speech-Icj-online-court.pdf [Fecha de consulta: 13 de marzo de 2020].

CORTÉS, P.; TAKAGI, T. (2019). «The Civil Money Claim Online: The Flagship Project of Court Digitalization in England and Wales». Computer and Telecommunications Law Review, núm. 25(8).

CORTÉS, P. (2018). «Using Technology and ADR Methods to Enhance Access to Justice». International Journal of Online Dispute Resolution, núm. 1-2, págs. 103-121.

HOUSE OF COMMONS SELECT JUSTICE COMMITTEE (2019). Court and Tribunal Reforms [en línea]. https://www.parliamentlive.tv/Event/Index/9f5ba45a-e4f0-485d-9697-b60e9ae15576 [Fecha de consulta: 13 de marzo de 2020].

KATSH, E.; RIFKIN J.; GAITENBY, A. (2000). «E-Commerce, E-Disputes, and E-Dispute Resolution: In the Shadow of "eBay Law"». Ohio State Journal on Dispute Resolution, vol. 15(3), pág. 727.

PALOMAR OLMEDA, A. (2012). «La actuación judicial automatizada». En: E. GAMERO CASADO y J. VALERO TORRIJOS (coords.). Las tecnologías de la información y la comunicación en la Administración de justicia: análisis sistemático de la Ley 18/2011, de 5 de julio. Navarra: Thomson Reuters-Aranzadi. Cizur Menor, pág. 481.

RULE, C. (2008). «Making Peace on eBay» [en línea]. ACResolution. http://colinrule.com/writing/ acr2008.pdf [Fecha de consulta: 13 de marzo de 2020].

SHEPPARD, C. (2018). The Traffic Penalty Tribunal [en línea]. https://assets.publishing.service.gov.uk/ government/uploads/system/uploads/attachment_data/file/762201/TECHNOLOGY_PLATFORMS_ KEY_NOTE_PRESENTATION_CAROLINE_.pdf [Fecha de consulta: 13 de marzo de 2020].

SLATER, S. (2017). Online dispute resolution and justice system integration. British Columbia's civil resolution tribunal [en línea]. https://wyaj.uwindsor.ca/index.php/wyaj/article/view/5008/4272 [Fecha de consulta: 13 de marzo de 2020].

VILALTA NICUESA, A. E. (2013). Mediación y arbitraje electrónicos. Navarra: Aranzadi. Cizur Menor, págs. 65-68. 


\title{
Cita recomendada
}

CATALÁN CHAMORRO, María José (2020). «El proceso judicial electrónico y su encaje en el ordenamiento jurídico español: estudio comparado con el proceso electrónico británico». IDP. Revista de Internet, Derecho y Política. N. ${ }^{\circ}$ 31, págs. xx-xx. UOC [Fecha de consulta: dd/mm/aa] http://dx.doi. org/10.7238/idp.v0i31.3220

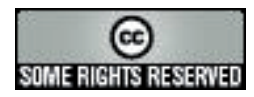

\begin{abstract}
Los textos publicados en esta revista están -si no se indica lo contrario- bajo una licencia Reconocimiento-Sin obras derivadas 3.0 España de Creative Commons. Puede copiarlos, distribuirlos y comunicarlos públicamente siempre que cite su autor y la revista y la institución que los publica (IDP. Revista de Internet, Derecho y Política; UOC); no haga con ellos obras derivadas. La licencia completa se puede consultar en: http://creativecommons.org/ licenses/by-nd/3.0/es/deed.es.
\end{abstract}

\section{Sobre la autora \\ María José Catalán Chamorro \\ Universidad de Córdoba \\ maria.jose.catalan@uco.es}

María José Catalán es doctora por la Universitat de València, donde realizó su tesis doctoral financiada por una ayuda predoctoral FPI-MINECO. Actualmente es profesora sustituta interina del área de Derecho Procesal de la Universidad de Córdoba y está acreditada como ayudante doctora por ANECA desde el año 2018. Se ha formado en las mejores universidades europeas, realizando diversas estancias de investigación en la Universidad de Dublín (Irlanda) o en la Universidad de Leicester (el Reino Unido), contando además con dos posgrados internacionales. Autora de un gran número de trabajos relacionados siempre con la protección del consumidor y su tutela judicial editados como capítulos de libros o artículos en revistas indexadas en numerosas bases de datos, ha publicado también un libro monográfico -Acceso a la Justicia de los consumidores a través del ADR y del ODR- del que ya se han vendido más de mil ejemplares. Asimismo, colabora con las Cortes Generales como experta para la redacción y modificación de leyes en materia de consumo y con la Generalitat de València en la redacción de la Ley de Mediación.

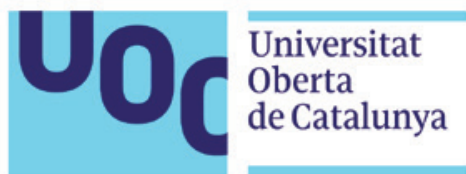

